\title{
Network Congestion-aware Online Service Function Chain Placement and Load Balancing
}

\author{
Xiaojun Shang \\ Stony Brook University \\ xiaojun.shang@stonybrook.edu
}

\author{
Zhenhua Liu \\ Stony Brook University \\ zhenhua.liu@stonybrook.edu
}

\author{
Yuanyuan Yang \\ Stony Brook University \\ yuanyuan.yang@stonybrook.edu
}

\begin{abstract}
Emerging virtual network functions (VNFs) introduce new flexibility and scalability into traditional middlebox. Specifically, middleboxes are virtualized as software-based platforms running on commodity servers known as network points of presence (N-PoPs). Traditional network services are therefore realized by chained VNFs, i.e., service function chains (SFCs), running on potentially multiple N-PoPs. SFCs can be flexibly placed and routed to reduce operating cost. However, excessively pursuing low cost may incur congestion on some popular $\mathrm{N}$-PoPs and links, which results in performance degradation or even violation of the service level of agreements.

In this paper, we first propose an optimization problem for joint SFC placement and routing. Given the problem is NP-hard, we design an approximation algorithm named candidate path selection (CPS) with a theoretical performance guarantee. We then propose an online optimization problem for placement of SFCs with fast demand fluctuation. The problem concerns migration costs of VNFs between time slots, and we design an online candidate path selection (OCPS) algorithm to handle it. Extensive simulation results highlight that the CPS and OCPS algorithms provide efficient placement and routing of SFCs comparable to the optimal solution.
\end{abstract}

\section{KEYWORDS}

cloud computing, virtual network function, service function chain, network congestion, load balancing.

\section{INTRODUCTION}

The development of virtual network functions (VNFs) migrates middleboxes (e.g., Proxies, Firewalls, Load Balancers) from dedicated hardware to commercial servers. Groups of these commercial servers placed at different locations are called network points of presence (N-PoPs) [11]. In this way, a network service can be converted to a service function chain (SFC) consisted of VNFs running on possibly multiple N-PoPs connected by a communication network. SFCs bring new flexibility which enables the reduction of the operating cost of middlebox networks.

ETSI standard [4] and several existing software-based VNF platforms $[13,19]$ highlight that different types of VNFs may demand different resources in the servers and some may require specific licenses to operate. Therefore, the operating cost of a certain type of VNFs heavily depends on the N-PoPs picked. This is especially

Permission to make digital or hard copies of all or part of this work for personal or classroom use is granted without fee provided that copies are not made or distributed for profit or commercial advantage and that copies bear this notice and the full citation on the first page. Copyrights for components of this work owned by others than ACM must be honored. Abstracting with credit is permitted. To copy otherwise, or republish, to post on servers or to redistribute to lists, requires prior specific permission and/or a fee. Request permissions from permissions@acm.org.

ICPP 2019, August 5-8, 2019, Kyoto, Japan

(C) 2019 Association for Computing Machinery.

ACM ISBN 978-1-4503-6295-5/19/08 ..\$15.00

https://doi.org/10.1145/3337821.3337850 the case for hybrid N-PoPs consisted of both commercial servers and dedicated hardware [17]. Thus, it is not surprising to see some previous work proposes to place VNFs over N-PoPs with the lowest cost to reduce the operating expenditure.

However, the operating cost of VNFs is not the only consideration in the placement of SFCs. In particular, the load on each N-PoP and network link should be carefully balanced to reduce congestion. Simply using constraints to limit the maximum load on N-PoPs and links, as most existing work does[6, 9], is not enough. Consider a simple example with two N-PoPs and two VNFs where each VNF requests for half of the resources of one N-PoP. Clearly, placing one VNF on an N-PoP each may bring much better performance compared to placing both VNFs on the same N-PoP, even though both placements satisfy the constraint. In other words, placing VNFs on the cheapest N-PoPs may incur congestion. Therefore, a thoughtful tradeoff between reducing operating cost and minimizing $\mathrm{N}-\mathrm{PoP} /$ network congestion is needed.

Furthermore, the placement of SFCs involves both placement of VNFs and routing of flows among VNFs because the placement of VNFs determines the sources and destinations for network flows. Jointly optimizing VNF placement and flow routing is crucial when considering congestion of both N-PoPs and links. For instance, there exist N-PoPs with sufficient VNF resources but limited bandwidth on some of its connecting links. In this way, placing VNFs on these $\mathrm{N}$-PoPs without jointly considering flow routing can easily aggregate large congestion on their connecting links with limited bandwidth. Existing work concerning network congestion either focuses on only one aspect $[1,12,19,20]$ or considers VNF placement and flow routing as two separate components [5], which results in sub-optimal solutions. Recent work [7] considers a predefined set of routes, which reduces the complexity at the cost of optimality.

The goal of this paper is to jointly optimize the SFC placement and routing to minimize the operating cost and congestion. The problem formulated based on this goal is challenging because one VNF cannot be split and placed on multiple N-PoPs, which leads to integer constraints. In addition, the requirement of jointly placing VNF and routing flows makes the problem even harder. Related work [12] proves the NP-hardness of jointly placing VNFs and routing flows when balancing load on links, thus proposing heuristic algorithms to solve it. In this paper, we propose an approximation algorithm solving the formulated problem in polynomial time with theoretical performance guarantee to the optimum.

Our SFC placement algorithm works well for such cases when workload demands of SFC are constant or change slowly. When there is a significant demand change, the algorithm is updated and SFCs are redeployed. This brings up a problem when demands fluctuate very fast, where the costs of redeploying SFCs (e.g., migrating VNFs) become comparable to or even higher than the operating costs. In this case, the SFC placement algorithm is modified to take the migration costs into consideration. In preceding research fields such as virtual 
network embedding (VNE), VM migration is an exorbitant procedure with high cost and large latency. However, some realized softwarebased VNF platforms such as ClickOS make the cost of VNF migration more affordable [13]. As a result, it is possible to pay moderate migration costs to reduce the operating cost and congestion when such migrations bring larger reductions. In this way, the problem becomes an online optimization, and we extend our algorithm to handle the lack of future information.

Our main contributions in this paper are summarized as follows.

- We formulate an optimization problem for the joint SFC placement and routing to minimize the operating cost and congestion. The problem bridges the operating cost and the network congestion which are separately considered in existing work.

- As the optimization problem involves integer linear programming (ILP), which is NP-hard, we propose an approximation algorithm named candidate path selection (CPS). The algorithm achieves bounded approximation ratio of $O(\log (|V|))$, where $|V|$ is the number of N-PoPs in the network. The approximation ratio is the cost of our algorithm compared to the optimal cost in the worst instance.

- When demands of SFCs fluctuate rapidly, we extend our algorithm into the online candidate path selection (OCPS) algorithm by optimizing over a time horizon with VNF migrations allowed between consecutive time slots with migration costs added to the objective function. OCPS approaches an competitive ratio of $\left[1+\Omega\left(\frac{1}{w}\right)\right] \cdot O(\log (|V|))$ compared to the offline optimum when the migration costs are small. Here, $w$ is the size of the prediction window. When the migration costs are relatively large, OCPS maintains good performance as shown in simulations.

- We perform extensive simulations to validate that our CPS algorithm achieves comparable performance to the optimal solution, which outperforms simpler baselines significantly. We also evaluate the performance of our online algorithm. When the demands of SFCs follow real traces with fast fluctuation, our OCPS algorithm is shown to maintain comparable performance to the offline optimum with comparable or even large migration costs than the operating costs.

The remainder of this paper is organized as follows. Section 2 presents the SFC placement problem and the CPS algorithm. Section 3 proposes the online SFC placement problem with fast demand fluctuation and the OCPS algorithm. Section 4 demonstrates the performance evaluation results and analysis. Section 5 briefly reviews the related work. Section 6 concludes this paper.

\section{SFC PLACEMENT WITH SLOW DEMAND FLUCTUATION}

Service function chains are much more flexible than traditional network services since a VNF can be placed on any capable N-PoP in the network with connecting links for the routing flows. Therefore, detailed SFC placement involves both the placement of VNFs and the routing of flows. Fig. 1 presents an example of SFC placement. The detailed placement is affected by many factors, e.g., operating cost, network congestion, demand fluctuation, security.

In this section, we focus on the operating cost and the network congestion to formulate the SFC placement problem. As mentioned in Section 1, pursuing low operating cost may lead to aggregation of load on some N-PoPs and links and thus cause congestion. On the

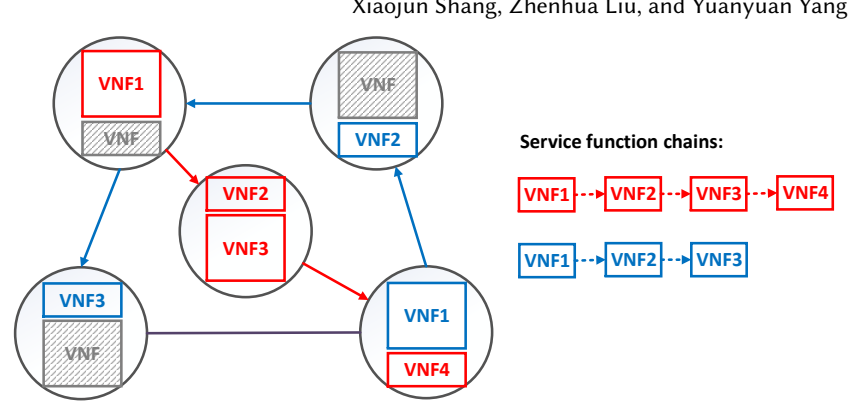

Figure 1: An example of SFC placement. The red and blue SFCs are deployed on to five N-PoPs. The sizes of VNFs represent different operating costs of VNFs. The colored arrows denote directions of routing between different N-PoPs. Routing paths between two VNFs may consist of multiple N-PoPs and physical links.

other side, reducing congestion requires distributing workload into the network evenly which moves some VNFs away from N-PoPs with the lowest operating cost. This natural tradeoff makes it important to consider the operating cost and the congestion jointly. In this way, in situations when computing resources and link bandwidth are sufficient, we can further reduce operating costs. Meanwhile, in situations when resources or bandwidth are limited, we can reduce latency and packet loss by avoiding severe congestion.

We formulate the following optimization framework to minimize the combination of operating cost and congestion cost. The model optimizes VNF placement and flow routing jointly. All notations used in this model are listed in Table 1.

Table 1: Notations

\begin{tabular}{|l|l|}
\hline Notation & Definition \\
\hline$V$ & set of all N-PoPs in the network \\
\hline$E$ & set of all connecting links between N-PoPs \\
\hline$X_{r}$ & set of all SFCs to be deployed into the network \\
\hline$x$ & set of VNFs demanded by SFC $r$ \\
\hline$A_{r, x}^{v}$ & $x^{t h}$ type of VNF in set $X_{r}$ \\
\hline$f_{r, x}^{e}$ & $\begin{array}{l}\in[0,1\}, \text { indicating whether VNF } x \text { of SFC } r \text { in placed on N-PoP } v \\
\text { from VNF } x \text { to the next VNF on SFC } r\end{array}$ \\
\hline$G(A, L)$ & operating cost of VNFs, determined by $A:\left\{A_{r, x}^{v}\right\}$ and $L:\left\{l_{r}\right\}$ \\
\hline$\alpha_{r, x}^{v}$ & the unit operating cost of VNF $x$ from SFC $r$ on N-PoP $v$ \\
\hline$\beta$ & cost parameter of the N-PoP congestion \\
\hline$\gamma$ & cost parameter of the link congestion \\
\hline$Y$ & maximal congestion ratio of N-PoPs \\
\hline$Z$ & maximal congestion ratio of links \\
\hline$i n(v)$ & set of links going into N-PoP $v$ \\
\hline$o u t(v)$ & set of links going out of N-PoP $v$ \\
\hline$l_{r}$ & service demand of SFC $r$ \\
\hline$M^{v}$ & capacity of N-PoP $v$ \\
\hline$N^{e}$ & bandwidth of link $e$ \\
\hline$v l_{r, x}^{e}$ & virtual link: link $e$ carrying flow from VNF $x$ to $x+1$ on SFC $r$ \\
\hline$v g_{r}$ & virtual graph consisting of virtual links for the same $r$ \\
\hline$\eta_{r, k}$ & the $k^{t h}$ virtual path consisting of virtual links of $r$ from source to sink \\
\hline$P r\left(\eta_{r, k}\right)$ & probability of virtual path $\eta_{r, k}$ \\
\hline$c p_{r, g}$ & $g^{t h}$ candidate path merged by all $\eta_{r, k}$ with the same VNF placement \\
\hline$P r\left(c p_{r, g}\right)$ & probability of picking candidate path $c p_{r, g}$ as placement of $r$ \\
\hline
\end{tabular}

\subsection{Optimization Model}

Denote the set of SFCs in the network as set $R$. SFC $r$ is an SFC in set $R$ with demand $l_{r}$, the amount of traffic load on $r . X_{r}$ is the set of VNFs on SFC $r$. We define two types of decision variables, $A_{r, x}^{v}$ 
and $f_{r, x}^{v}$, for the joint VNF placement and routing. $A_{r, x}^{v}$ is a binary variable which indicates whether VNF $x$ on SFC $r$ is placed at N-PoP $v \in V$, where $V$ is the set of all N-PoPs in the network. $f_{r, x}^{e} \in[0,1]$ denotes the fraction of flow routing from VNF $x$ to the next VNF $x+1$ of SFC $r$ on link $e \in E$, where $E$ is the set of all links in the network.

Operating cost: Denote by $\mathcal{G}(A, L)$ as the operating cost of VNFs, where $A=\left\{A_{r, x}^{v}\right\}$ and $L=\left\{l_{r}\right\} . \mathcal{G}$ is a linear function determined by $A$ and $L$ which can be extended to different forms based on actual conditions. In the following sections of this paper, without loss of generality, we define an $\alpha_{r, x}^{v}$ as the operating cost of deploying one unit of VNF $x$ from SFC $r$ on N-PoP $v \in V$. Therefore, we restrict $\mathcal{G}(A, L)$ as $\sum_{r \in R} \sum_{x \in X_{r}} \sum_{v \in V} \alpha_{r, x}^{v} \cdot l_{r} \cdot A_{r, x}^{v}$.

$N-P o P$ congestion: Denote by $M^{v}$ the capacity of N-PoP $v$. We define $y^{v}$ as the congestion ratio of N-PoP $v$, which indicates the overall load on $v$ divided by $M^{v}$. We have $y^{v}=\sum_{r \in R} \sum_{x \in X_{r}} \frac{l_{r}}{M^{v}} \cdot A_{r, x}^{v}$. The congestion ratio of an N-PoP represents the utilization of this $\mathrm{N}-\mathrm{PoP}$ and how much latency and packet loss it may introduce into the network. Denote by $Y$, a non-negative variable, the maximum congestion ratio of all N-PoPs. we have $Y=\max \left\{y^{v} \mid v \in V\right\}$. Note that $Y$ could be larger than 1, which is easily handled by mechanisms such as resource sharing or queuing. However, since the total amount of VNFs to be deployed into the network is fixed, the larger $Y$ is, the more congested N-PoPs and congestion cost we will have. We introduce $\beta$ as the cost parameter of the N-PoP congestion. The cost of N-PoP congestion in the network is thus denoted as $\beta \cdot Y$.

Link congestion: Similarly, $N^{e}$ is the bandwidth of physical link e. $z^{e}$ is the congestion ratio of link e and $z^{e}=\sum_{r \in R} \sum_{x \in X_{r}} \frac{l_{r}}{N^{e}} \cdot f_{r, x}^{e}$. Denote by $Z$ the maximum congestion ratio of links, $Z=\max \left\{z^{e} \mid e \in\right.$ $E$ \}. Denote $\gamma$ as the cost parameter of the link congestion and we have $\gamma \cdot Z$ as the cost of link congestion.

The relative sizes of $\beta$ and $\gamma$ compared to $\mathcal{G}$ are determined by the actual requirements and conditions. For instance, $\beta$ and $\gamma$ should be relatively large if the network services require low latency (e.g., online games, live broadcast) or the N-PoPs have no waiting queues and drop packets whenever congested.

With above definitions, the SFC placement model is formulated as follows.

$$
\begin{array}{lll}
\min & \mathcal{G}(A, L)+\beta \cdot Y+\gamma \cdot Z & \\
\text { s.t. } & & \\
& \sum_{v \in V} A_{r, x}^{v}=1 \quad \forall r \in R, \forall x \in X_{r} \\
& \sum_{e \in \text { in }(v)} f_{r, x}^{e}-\sum_{e \in \text { out }(v)} f_{r, x}^{e}=A_{r, x+1}^{v}-A_{r, x}^{v} \\
& \forall r \in R, \forall x \in X_{r}, \forall v \in V & \\
& \sum_{r \in R} \sum_{x \in X_{r}} \frac{l_{r}}{M^{v}} \cdot A_{r, x}^{v} \leq Y & \\
& \sum_{r \in R} \sum_{x \in X_{r}} \frac{l_{r}}{N^{e}} \cdot f_{r, x}^{e} \leq Z & \\
& A_{r, x}^{v} \in\{0,1\}, \quad f_{r, x}^{e} \in[0,1] & \\
& \forall r \in R, \forall x \in X_{r}, \forall v \in V, \forall e \in E
\end{array}
$$

The objective is to minimize a linear combination of operating cost and network congestion cost. Constraint (1) indicates that each VNF on one SFC is placed exactly once. Constraint (2) makes sure that given the VNF placement, all flow routings are satisfied. If both or neither of VNF $x$ and $x+1$ is placed on the same N-PoP $v$, i.e., $A_{r, x+1}^{v}-A_{r, x}^{v}=0$, the in-flow and out-flow $f_{r, x}^{v}$ to node $v$ should be the same. On the other hand, if one of the two VNFs is placed on node $v$, the difference of in-flow and out-flow should be \pm 1 . Constraint (3) and (4) make sure that $Y$ and $Z$ are the maximum congestion ratio over N-PoPs and links. If the start and destination of each SFC are fixed, we can add constraints similar to Constraint (2) to regulate the start and end of each $r$. Otherwise, the start and destination of all SFCs are two abstract nodes connecting to all N-PoPs.

According to [12], the problem of jointly placing VNFs and routing flows minimizing congestion on links is NP-hard, which is a special case of our model when parameters $\alpha$ and $\beta$ are zeros. Therefore, the model formulated in this section also leads to an NP-hard problem. In the following section, we propose an approximation algorithm to solve the problem in polynomial time and yield SFC placement with bounded performance to the optimal solution. All notations used in the algorithm can be found in Table 1 .

\subsection{Candidate Path Selection Algorithm}

In this section, we propose an approximation algorithm with polynomial time complexity named Candidate Path Selection (CPS) to yield the scheme of SFC placement with an approximation ratio of $O(\log |V|)$, where $|V|$ is the total number of N-PoPs.

CPS first relaxes the original ILP problem into a linear programming (LP) problem by relaxing $A_{r, x}^{v} \in\{0,1\}$ into $A_{r, x}^{v} \in[0,1]$. It then solves the relaxed LP and gets the (fractional) solution $\left\{A_{r, x}^{v}\right\}$ and $\left\{f_{r, x}^{e}\right\}$. Define a virtual link $v l_{r, x}^{e}$ which represents the physical link $e$ carrying the flow from VNF $x$ to VNF $x+1$ on SFC $r$. Each virtual link $v l_{r, x}^{e}$ corresponds to one $f_{r, x}^{e}$. With the same $r$, all $v l_{r, x}^{e}$ with positive $f_{r, x}^{e}$ formulate a virtual graph $v g_{r}$. The algorithm then does the following process for each SFC $r$ to place it into the network.

In the virtual graph $v g_{r}$, CPS selects the virtual link $v l_{r, x^{\prime}}^{e^{\prime}}$ with the smallest positive $f_{r, x}^{e}$. Then, from the starting point of the selected virtual link, CPS finds the adjacent former virtual link pointing upward to the source of the SFC $r$. The former virtual link satisfies following conditions. First, it belongs to the virtual graph $v g_{r}$. Second, its ending point is the start point of $v l_{r, x^{\prime}}^{e^{\prime}}$. Third, the former virtual link has the largest $x$ which is smaller or equal to $x^{\prime}$. We call these conditions virtual path conditions (VPC). If multiple links satisfy VPC, CPS randomly picks one as the former virtual link. CPS keeps finding former virtual links until reaching the source of the SFC $r$. In a similar method, CPS finds latter virtual links until the sink of $r$ and a virtual path $\eta_{r, k}$ is thus created for the SFC from source to sink. All $f_{r, x}^{e}$ of the virtual links along the selected virtual path are subtracted by $f_{r, x^{\prime}}^{e^{\prime}}$ In this way, the selected virtual link $v l_{r, x^{\prime}}^{e^{\prime}}$ is removed from the virtual graph. The value of $f_{r, x^{\prime}}^{e^{\prime}}$ equals to $\operatorname{Pr}\left(\eta_{r, k}\right)$.

Based on $\eta_{r, k}$, CPS further figures out the placement of each VNF because virtual links contain information of both VNF placement and flow routing. Repeat picking $\eta_{r, k}$ until no positive $f_{r, x}^{e}$ left for $r$. Since all virtual paths with the same VNF placement can be seen as one SFC placement with multi-path routing, the algorithm merges all virtual paths $\eta_{r, k}$ which place their VNFs at same N-PoPs but route flows with different links as one candidate path $c p_{r, g}$. The probability $\operatorname{Pr}\left(c p_{r, g}\right)$ is the summation of all $\operatorname{Pr}\left(\eta_{r, k}\right)$ from the merged paths. The distributing of flow on each routing path is weighted by $\frac{\operatorname{Pr}\left(\eta_{r, k}\right)}{\operatorname{Pr}\left(c p_{r, g}\right)}$. In the way, CPS gets each SFC $r$ a candidate path set. Select $c p_{r, g}$ in 
the candidate path set as the actual SFC placement scheme according to the probability $\operatorname{Pr}\left(c p_{r, g}\right)$. This specific value of $\operatorname{Pr}\left(c p_{r, g}\right)$ is an important prerequisite for the proof of our theoretical performance guarantee which will be discussed in detail in following sections.

The detailed algorithm is shown in Algorithm 1. Line 3 to 12 describe how to find candidate paths. Line 13 to 19 illustrate how to place VNFs according to candidate paths. Line 20 to 24 merge candidate paths with the same VNF placement and select a candidate path for each SFC $r$ as its placement and routing scheme.

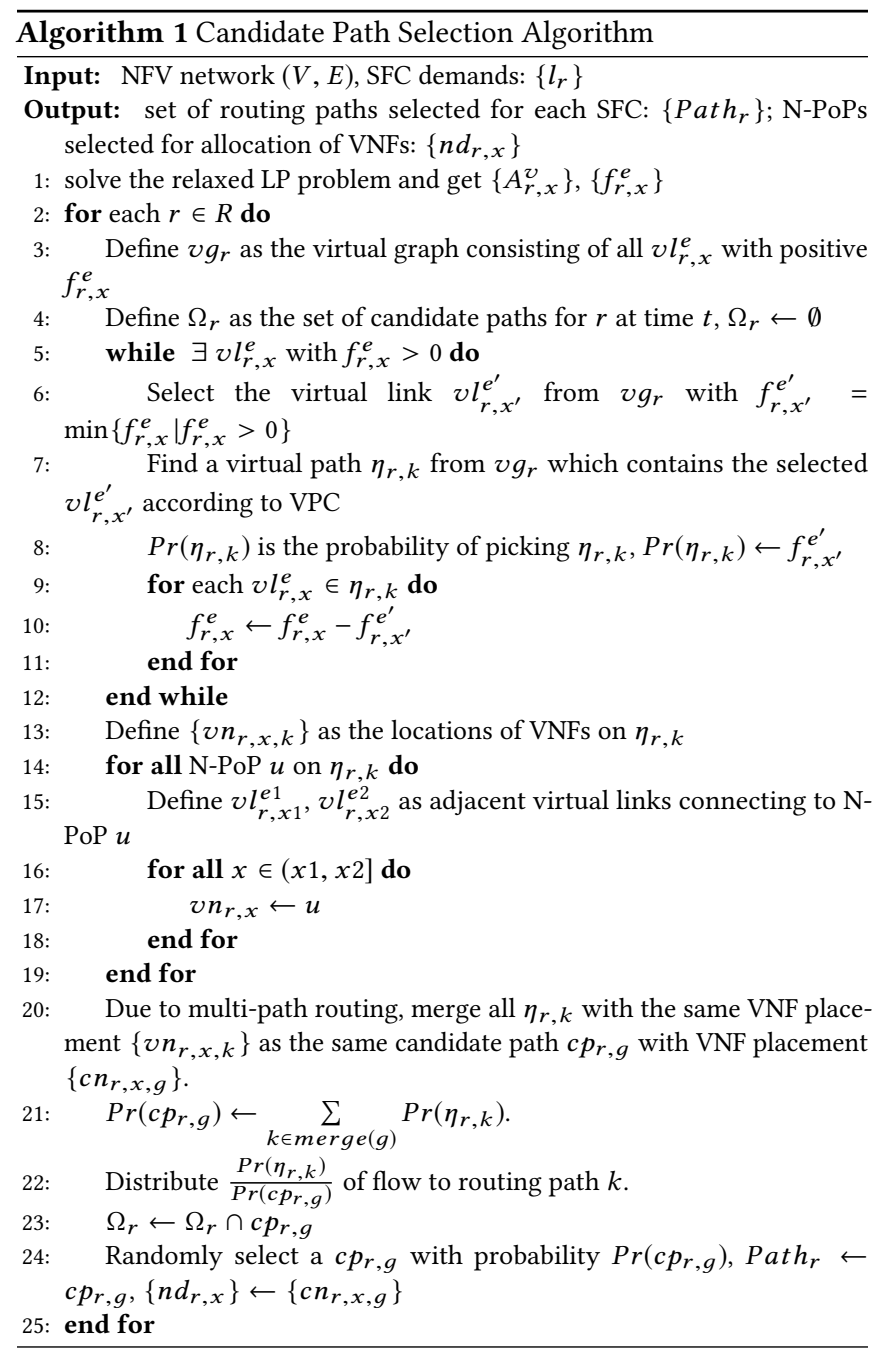

We now analyze the time complexity of Algorithm 1 besides solving an LP problem. For each SFC, Algorithm 1 iteratively seeks for the virtual link with the smallest positive $f_{r, x}^{e}$ and reduces it to zero. Hence, there are at most $\left|X_{r}\right||E|$ iterations before all $f_{r, x}^{e}$ are reduced to zero. Meanwhile, in each iteration, Algorithm 1 finds a virtual path and subtracts the value of each link on the candidate path by the smallest positive $f_{r, x}^{e}$ in at most $\left|X_{r}\right||E|$ iterations. According to the determined virtual paths, Algorithm 1 also allocates VNFs on N-PoPs in $\left|X_{r}\right||V|$ iterations. Since in general, the number of VNF types in the network is a small constant, $\left|X_{r}\right|$ can be omitted in the expression of complexity. There are a total of $|R|$ SFCs, so the computational complexity is $O\left(|R||E|^{2}\right)$. We then prove the theoretical bound of Algorithm 1 to the optimum solution.

\subsection{Proof of the Approximation Ratio}

The approximation ratio between the CPS algorithm and the optimal solution is $O(\log (|V|))$. This conclusion is summarized as Theorem 2.1. In the theorem, we denote $l_{\max }$ as the maximal $l_{r}$ and $l_{\min }$ as the minimal $l_{r}$. Similarly, $M_{\max }, M_{\min }, N_{\max }, N_{\min }, \alpha_{\max }$ and $\alpha_{\min }$ are corresponding maximum and minimum of $M^{v}, N^{e}$ and $\alpha_{r, x}^{v}$.

THeOREm 2.1. The total cost solved by Algorithm 1 guarantees with high probability an approximation ratio of $1+d \cdot \ln (|V|)$ to the optimal solution of the ILP problem, where

$d=\max \left\{\frac{\alpha_{\max } \cdot l_{\max }}{|R||X| \cdot \alpha_{\min } \cdot l_{\min }}, \frac{2 \cdot l_{\max } \cdot M_{\max }}{l_{\min } \cdot M_{\min }}, \frac{3 \cdot l_{\max } \cdot N_{\max }}{l_{\min } \cdot N_{\min }}, \mathrm{e}^{2}\right\}$.

To prove Theorem 2.1, we first introduce Lemma 2.2 and Lemma 2.3 .

Lemma 2.2. Suppose $\mathcal{X}_{1}, \mathcal{X}_{2}, \ldots, \mathcal{X}_{m}$ are binary independent random variables with $\operatorname{Pr}\left\{\mathcal{X}_{i}=1\right\}=p_{i}$ and $\operatorname{Pr}\left\{\mathcal{X}_{i}=0\right\}=1-p_{i}$ for each $i \in\{1,2, \ldots, m\}$. Let $\mathcal{Y}=\sum_{i} a_{i} \mathcal{X}_{i}$ with $a_{i} \geq 0$. $h$ and $\mathcal{Z}$ are positive real numbers with $h \geq \max \left\{a_{i} \mid i \in[1, m]\right\}$ and $\mathcal{Z} \geq \mathbb{E}(\mathcal{Y})$. For any $\Delta \geq 0$, we have $\operatorname{Pr}\{\mathcal{Y} \geq(1+\Delta) \mathcal{Z}\} \leq\left[\mathrm{e}^{\Delta}(1+\Delta)^{-\Delta-1}\right]^{\frac{Z}{h}}$.

The detailed proof of Lemma 2.2 can be found in [8].

LEMMA 2.3. For each SFC $r, \Theta_{r, x}^{v}$ is the set of candidate paths which allocate VNF $x$ on node $v$. The sum of all probabilities of the candidate paths in $\Theta_{r, x}^{v}$ equals to the probability that $v$ is chosen to deploy VNF $x$. Formally,

$$
\sum_{c p_{r, g} \in \Theta_{r, x}^{v}} \operatorname{Pr}\left(c p_{r, g}\right)=A_{r, x}^{v} .
$$

Lemma 2.3 is proved using the strong correlation of the fractional solution $A_{r, x}^{v}$ and $f_{r, x}^{e}$. Though the probabilities of virtual paths in Algorithm 1 are determined by $f_{r, x}^{e}$, they also agree with $A_{r, x}^{v}$, the solution of the VNF allocation. Thus, when the placement of virtual links is fixed, the allocation of VNFs is also determined. The details of the proof are omitted here due to limited space.

Given Lemma 2.2 and 2.3, we can prove the bound of the operating cost and the congestion cost to the optimum respectively, which leads to Lemma 2.4, 2.5 and 2.6.

Lemma 2.4. The operating cost solved by Algorithm 1 guarantees with high probability an approximation ratio of $1+a \cdot \ln (|V|)$ to $H$, the total operating cost from the LP solution, where

$$
a=\max \left\{\frac{\alpha_{\max } \cdot l_{\max }}{|R||X| \cdot \alpha_{\min } \cdot l_{\min }}, \mathrm{e}^{2}\right\} .
$$

Proof. For each VNF placement, we define a random variable $X_{r, x}^{v}$. If node $v$ is selected to map VNF $x$ of SFC $r, X_{r, x}^{v}=1$. Otherwise $X_{r, x}^{v}=0$. According to Lemma 2.3,

$$
\mathbb{E}\left(X_{r, x}^{v}\right)=\sum_{c p_{r, g} \in \Theta_{r, x}^{v}} \operatorname{Pr}\left(c p_{r, g}\right)=A_{r, x}^{v} .
$$

Next, consider random variable $\mathcal{Y}=\sum_{r \in R} \sum_{x \in X_{r}} \sum_{v \in V} \alpha_{x}^{v} \cdot l_{r} \cdot \mathcal{X}_{r, x}^{v}$ which represents the VNF operating cost under the approximation algorithm. Hence,

$$
\mathbb{E}(\mathcal{Y})=\sum_{r \in R} \sum_{x \in X_{r}} \sum_{v \in V} \alpha_{x}^{v} \cdot l_{r} \cdot A_{r, x}^{v} .
$$

which is also the operating cost of the relaxed LP problem denoted as $H$. Applying Lemma 2.2, we have

$$
\operatorname{Pr}(\boldsymbol{Y} \geq(1+\Delta) H) \leq\left[e^{\Delta}(1+\Delta)^{-\Delta-1}\right]^{\frac{H}{h}}
$$




$$
\leq\left[\left(\frac{\Delta}{e}\right)^{-\Delta}\right]^{\frac{H}{h}} .
$$

Recalling that $|V|$ is the number of nodes in the network, let $\Delta=$ $a \ln (|V|)$ and $h=a_{\text {max }} \cdot l_{\text {max }}$. Since $a=\max \left\{\frac{\alpha_{\text {max }} \cdot l_{\text {max }}}{|R||X| \cdot \alpha_{\text {min }} \cdot l_{\text {min }}}, e^{2}\right\}$, we derive that

$$
\begin{aligned}
\ln \left(\frac{\Delta}{e}\right)^{\Delta}= & a \ln (|V|)(\ln (a)+\ln \ln (|V|)-1) \geq a \ln (|V|) \\
& \geq \frac{\alpha_{\text {max }} \cdot l_{\text {max }}}{|R||X| \cdot \alpha_{\text {min }} \cdot l_{\text {min }}} \ln (|V|) .
\end{aligned}
$$

And further, $\left(\frac{\gamma}{e}\right)^{-\Delta} \leq|V|^{-\frac{\alpha_{\max } \cdot l_{\max }}{|R| X \mid \cdot \alpha_{\min } \cdot l_{\min }}}$. Clearly, $H \geq|R||X|$. $\alpha_{\min } \cdot l_{\min }$. Combining all the solutions above, we get

$$
\begin{aligned}
& \operatorname{Pr}\{\boldsymbol{y} \geq(1+\Delta) H\} \leq\left[|V|^{-\frac{\alpha_{\max } \cdot l_{\max }}{|T||R||X| \cdot \alpha_{\min } \cdot l_{\min }}}\right]^{\frac{H}{h}} \\
& \leq\left[|V|^{-\frac{\alpha_{\max } \cdot l_{\max }}{|R||X| \cdot \alpha_{\min } \cdot l_{\min }}}\right]^{\frac{|R \| X| \cdot \alpha_{\min } \cdot l_{\min }}{\alpha_{\max } \cdot l_{\max }}}=|V|^{-1} .
\end{aligned}
$$

We continue to present and prove Lemma 2.5. The proof shares similar components to the proof of Lemma 2.4. We only show the important differences below.

Lemma 2.5. The node congestion solved by Algorithm 1 guarantees with high probability an approximation ratio of $1+b \cdot \ln (|V|)$ to $J$, the node congestion cost from the LP solution, where

$$
b=\max \left\{\frac{2 \cdot l_{\max } \cdot M_{\max }}{l_{\min } \cdot M_{\min }}, \mathrm{e}^{2}\right\} .
$$

Proof. With the same definition of $X_{r, x}^{v}$, we now consider the random variable $\mathcal{Y}^{v}=\sum_{r \in R} \sum_{x \in X_{r}} \frac{l_{r}}{M^{v}} \cdot X_{r, x}^{v}$, which represents the congestion ratio on node $v$ from Algorithm 1. Hence,

$$
\mathbb{E}\left(\mathcal{Y}^{v}\right)=\sum_{r \in R} \sum_{x \in X_{r}} \frac{l_{r}}{M^{v}} \cdot A_{r, x}^{v}
$$

According to constraint 3, we have $\mathbb{E}\left(\boldsymbol{Y}^{v}\right) \leq J$. Applying Lemma 2.2, we have $\left.\operatorname{Pr}\left(\mathcal{Y}^{v}\right) \geq(1+\Delta) J\right) \leq\left[\left(\frac{\Delta}{e}\right)^{-\Delta}\right]^{\frac{J}{h}}$. Similarly, $\Delta=b$. $\ln (|V|), h=\frac{l_{\max }}{M_{\min }}$ and $b=\max \left\{\frac{2 \cdot l_{\max } \cdot M_{\max }}{l_{\min } \cdot M_{\min }}, e^{2}\right\}$, we have $\left(\frac{\Delta}{e}\right)^{-\Delta} \leq$ $|V|^{-\frac{l_{\max } \cdot M_{\max }}{l_{\min } \cdot M_{\min }} \text {. }}$

Within our application scenarios, we always have $|R| \cdot|X| \geq|V|$. In this way $J \geq \frac{|R| \cdot|X|}{|V|} \cdot \frac{l_{\min }}{M_{\max }} \geq \frac{l_{\min }}{M_{\max }}$. Combining all conclusions above, we have

$$
\begin{aligned}
& \operatorname{Pr}\left\{\boldsymbol{y}^{v} \geq(1+\Delta) J\right\} \leq\left[|V|^{-\frac{2 \cdot l_{\max } \cdot M_{\max }}{l_{\min } \cdot M_{\min }}}\right]^{\frac{J}{h}} \\
& \leq\left[|V|^{-\frac{2 \cdot l_{\max } \cdot M_{\max }}{\mid T \cdot \cdot l_{\min } \cdot M_{\min }}}\right]^{\mid \frac{|T| \cdot l_{\min } \cdot M_{\min }}{l_{\max } \cdot M_{\max }}}=|V|^{-2} \text {. }
\end{aligned}
$$

According to the Boole's inequality,

$$
\begin{gathered}
\operatorname{Pr}\left\{\exists v \in V: \mathcal{y}^{v} \geq(1+\Delta) J\right\} \\
\leq|\operatorname{V}| \operatorname{Pr}\left\{\boldsymbol{y}^{v} \geq(1+\Delta) J\right\} \leq|V| \cdot|V|^{-2}=|V|^{-1} .
\end{gathered}
$$

We then present Lemma 2.6 and show the proof.

Lemma 2.6. The link congestion solved by Algorithm 1 guarantees with high probability an approximation ratio of $1+c \cdot \ln (|V|)$ to $K$, the link congestion cost from the LP solution, where

$$
c=\max \left\{\frac{3 \cdot l_{\max } \cdot N_{\max }}{l_{\min } \cdot N_{\min }}, \mathrm{e}^{2}\right\} .
$$

Proof. We now consider $\mathcal{Y}^{e}$, the congestion ratio on link $e$ from Algorithm 1. In this proof, $\mathcal{X}_{r, x}^{e}=1$ represents that there is a flow on link $e$ from VNF $i$ to the next VNF on SFC $r$. Due to the multi-path routing, we have

$$
y^{e} \leq \sum_{r \in R} \sum_{x \in X_{r}} \frac{l_{r}}{N^{e}} \cdot X_{r, x}^{e}
$$

Then, we have

$$
\mathbb{E}\left(\boldsymbol{y}^{e}\right) \leq \sum_{r \in R} \sum_{x \in X_{r}} \frac{l_{r}}{N^{e}} \cdot f_{r, x}^{e} \leq K
$$

In this way, Lemma 2.2 is still applicable. Following a similar proof of Lemma 2.5, we can get

$$
\begin{gathered}
\operatorname{Pr}\left\{\exists e \in E: \mathcal{Y}^{e} \leq|V|^{2} \operatorname{Pr}\left\{y^{e} \geq(1+\Delta) K\right\}\right. \\
\leq|V|^{2} \cdot|V|^{-3}=|V|^{-1} .
\end{gathered}
$$

With all Lemmas 3, 4, 5 proved, we finally prove Theorem 2.1.

Proof. We define a positive number $d$, where $d=\max \left\{a, b, c, e^{2}\right\}$. We also represent the overall cost solved by Algorithm 1 as $\operatorname{Cost}\left(A L G_{1}\right)$ and the optimal cost of the ILP problem as OPT. With Lemma 2.4, 2.5 and 2.6, we conclude that

$$
\operatorname{Cost}\left(A L G_{1}\right) \leq(1+d \cdot \ln (|V|)) \cdot(J+H+K)
$$

with high probability. Meanwhile, $J+H+K$ is the solution of the LP and serves as the lower pound of the optimal solution of the ILP that $J+H+K \leq O P T$. In this way, we have

$$
\operatorname{Cost}\left(A L G_{1}\right) \leq(1+d \cdot \ln (|V|)) \cdot O P T .
$$

With the proof, we conclude that the cost of Algorithm 1 possesses bounded approximation ratio of $O(\log (|V|))$ to the optimal solution with high probability.

\section{ONLINE SFC PLACEMENT WITH FAST DEMAND FLUCTUATION}

There exist scenarios when demands of SFCs fluctuate very fast. In these cases, migration costs of VNFs become comparable to the operating costs, because the operating costs of VNFs are now counted within short time slots. Therefore, simply applying the SFC placement algorithm in Section 2 whenever a demand changes is no longer appropriate. Since the different placement of VNFs between adjacent time slots may introduce large migration costs. According to realized VNF platforms [19] [13], cost of VNF migration can be much lower than that of VM migration. In this way, it is possible to reduce the total cost by paying moderate migration cost for lower operating cost and congestion. When considering migration costs of VNFs, the original problem becomes an online SFC placement problem seeking for the minimized total cost of VNF operating, congestion and VNF migration over the entire time span $T$. We formulate the online optimization framework in the following section to characterize this problem. 


\subsection{Online Optimization Model}

In this section, we introduce a dimension $t$ and define the service demand of SFC $r$ at time $t$ as $l_{r}(t)$. Therefore, the fluctuation of demands is represented by different $l_{r}(t)$ at different time $t$. Furthermore, all $l_{r}(\tau)$ with $\tau>t$ are unknown at $t$. Similar to the model in Section 2, define online decision variables as $A_{r, x}^{v}(t)$ and $f_{r, x}^{e}(t)$, which represent the VNF placement and flow routing at time $t$. Denote by $\mathcal{G}[A(t), L(t)]$ the operating cost, where $A(t)=\left\{A_{r, x}^{v}(t)\right\}$ and $L(t)=\left\{l_{r}(t)\right\}$. Define $Y(t)$ and $Z(t)$ as the maximal congestion cost on N-PoPs and links at time $t$.

Migration cost: The migration cost in the objective function is a major difference between the online model and that in Section 2. Denote by $\delta\|A(t)-A(t-1)\|$ the migration cost of VNFs, where $\delta \in \mathbb{R}^{+}$and $\|$.$\| can be any norm in \mathbb{R}^{n}$. In this paper, without loss of generality, we define $\delta_{r, x}$ as the migration cost of VNF $x$ on SFC $r$ and assume that migrating a VNF in and out costs the same. In this way, the migration cost at time $t$ is $\sum_{r \in R} \sum_{x \in X_{r}} \sum_{v \in V} \delta_{r, x} \cdot \mid A_{r, x}^{v}(t)-$ $A_{r, x}^{v}(t-1) \mid$.

The relative values of $\mathcal{G}$ and $\delta_{r, x}$ are affected by the length of a time slot and the type of a VNF. If VNF demands change fast with very short time slots or a type of VNF involves large data migration and long latency to migrate, $\delta_{r, x}$ should be relatively large.

The online SFC placement model is thus formulated as follows.

$$
\begin{array}{ll}
\min \quad & \sum_{t \in T}\{\mathcal{G}[A(t), L(t)]+\beta \cdot Y(t)+\gamma \cdot Z(t) \\
& +\delta\|A(t)-A(t-1)\|\} \\
\text { s.t. } \quad & \sum_{v \in V} A_{r, x}^{v}(t)=1 \\
& \forall t \in T, \forall r \in R, \forall x \in X_{r} \\
& \sum_{e 1 \in i n(v)} f_{r, x}^{e 1}(t)-\sum_{e 2 \in o u t(v)} f_{r, x}^{e 2}(t)= \\
& A_{r, x+1}^{v}(t)-A_{r, x}^{v}(t) \\
& \forall t \in T, \forall r \in R, \forall x \in X_{r}, \forall v \in V \\
& \sum_{r \in R} \sum_{x \in X_{r}} \frac{l_{r}(t)}{M^{v}} \cdot A_{r, x}^{v}(t) \leq Y(t) \\
& \forall t \in T, \forall v \in V \\
& \sum_{r \in R} \sum_{x \in X_{r}} \frac{l_{r}(t)}{N^{e}} \cdot f_{r, x}^{e}(t) \leq Z(t) \\
& \forall t \in T, \forall e \in E \\
& A_{r, x}^{v}(t) \in\{0,1\}, \quad f_{r, x}^{e}(t) \in[0,1] \\
& \forall t \in T, \forall r \in R, \forall x \in X_{r}, \forall v \in V, \forall e \in E
\end{array}
$$

The formulated model leads to an online integer optimization problem with unknown information of $\left\{l_{r}(\tau) \mid \tau \in[t, T]\right\}$ at any time $t-1$. This model leads to a general case of the problem we discussed in Section 2 which is already NP-hard. Therefore, we propose the online candidate path selection (OCPS) algorithm to solve it in polynomial time.

At each time $t-1$, OCPS yields the placement of SFCs for time $t$ in two steps. In the first step, the algorithm relaxes the online integer optimization problem into an online convex optimization (OCO) problem $\left(A_{r, x}^{v}(t)\right.$ from $\{0,1\}$ to $\left.[0,1]\right)$ and applies the method of averaging fixed horizon control (AFHC) proposed in our previous work [10] to solve the relaxed problem. The (fractional) result of AFHC has bounded competitive ratio to the offline optimum (integral). In the second step, OCPS applies an online candidate selection method which gets each SFC $r$ a set of candidate paths based on both the solution of AFHC and the placement of SFC $r$ at time $t-1$. It then picks one candidate path as the placement of $r$ for time $t$ according to a novel criterion.

In the following two sections, we first illustrate AFHC used in the first step and then present the novel online candidate path selection method in the second step.

\subsection{Averaging Fixed Horizon Control}

AFHC proposed in our previous work [10] is a promising method to solve OCO problems which is proactive and relies on predictions of future demands. The prediction can be done by methods such as machine learning which is beyond our concern in this paper. Different from previous horizon control methods such as receding horizon control (RHC) [14], AFHC makes decisions based on not only predictions but also preceding data. Our online SFC placement algorithm takes advantage of this feature to prevent unnecessary VNF migration between adjacent time slots. AFHC is proved to have a competitive ratio of $1+O\left(\frac{1}{w}\right)$ to the offline optimal in the perfect lookahead model [10] and a strong performance guarantee in cases of noisy predictions [2]. Detailed AFHC applied in our algorithm is summarized as follows.

At each time $t-1$, we determine the decision variables for the upcoming time $t$ using AFHC. For each $t-1$, we have a prediction window of size $w$. We use $\left\{l_{r}^{*}(\tau) \mid \tau \in[t, t+w-1]\right\}$ to represent the predictions made at time $t-1$ which contains future SFC demands in $[t, t+w-1]$. We then solve the OCO problem over the time span $[t, t+w-1]$ using the predictions in polynomial time. Solutions are represented by $\left\{A_{r, x}^{* v}(\tau) \mid \tau \in[t, t+w-1]\right\}$ and $\left\{f_{r, x}^{* e}(\tau) \mid \tau \in[t, t+w-\right.$ $1]\}$. We pick out variables for time $t$ in these solutions and denote them as sets $\left\{\mathcal{A}_{r, x}^{t-1, v} \mid \mathcal{A}_{r, x}^{t-1, v}=A_{r, x}^{* v}(t)\right\}$ and $\left\{\mathcal{F}_{r, x}^{t-1, e} \mid \mathcal{F}_{r, x}^{t-1, e}=\right.$ $\left.f_{r, x}^{e}(t)\right\}$. The header $t-1$ on $\mathcal{A}_{r, x}^{t-1, v}$ means the variable is solved by the predictions made at time $t-1$. With similar processes, we get sets $\left\{\mathcal{A}_{r, x}^{\tau, v} \mid \tau \in[t-w, t-2]\right\}$ and $\left\{\mathcal{F}_{r, x}^{\tau, e} \mid \tau \in[t-w, t-2]\right\}$ using predictions made at time $\tau$. The only difference is that all $\left\{l_{r}^{*}(\tau) \mid \tau \leq t-1\right\}$ in the predictions are replaced by real value $l_{r}(\tau)$ since they are already known at time $t-1$. According to AFHC, the decision variables at time $t$ are thus determined as $A_{r, x}^{v}(t)=\frac{1}{w} \cdot \sum_{\tau=t-w}^{t-1} \mathcal{A}_{r, x}^{\tau, v}$ and $f_{r, x}^{v}(t)=\frac{1}{w} \cdot \sum_{\tau=t-w}^{t-1} \mathcal{F}_{r, x}^{\tau, e}$.

\subsection{Online Candidate Path Selection Method}

Considering migration cost, applying the candidate path selection method in Algorithm 1 directly based on the result of AFHC is not applicable. The ratio between the output and the optimum is not bounded with counterexamples. The reason is that the expected migration cost in Algorithm 1 can be larger than the fractional result from AFHC. In other words, there exist situations when

$$
\begin{aligned}
& \mathbb{E}\left[\sum_{t \in T} \sum_{r \in R} \sum_{x \in X_{r}} \delta_{r, x} \cdot\left|X_{r, x}^{v}(t)-X_{r, x}^{v}(t-1)\right|\right] \\
& >\sum_{t \in T} \sum_{r \in R} \sum_{x \in X_{r}} \delta_{r, x} \cdot\left|A_{r, x}^{v}(t)-A_{r, x}^{v}(t-1)\right| .
\end{aligned}
$$

Here, we denote $X_{r, x}^{v}(t)$ as the binary variable determining VNF placement from Algorithm 1 and $A_{r, x}^{v}(t)$ as the corresponding relaxed variable in AFHC. Therefore, the key part in the proof of Theorem 2.1 does not hold and the migration cost can be arbitrarily larger than the AFHC result. We further explain this phenomenon using an 
counterexample. Suppose that we have $A_{r, x}^{v_{1}}(t)=A_{r, x}^{v_{1}}(t-1)=0.5$ and $A_{r, x}^{v_{2}}(t)=A_{r, x}^{v_{2}}(t-1)=0.5$. So the migration cost of placing VNF $x$ on SFC $r$ within $[t-1, t]$ is 0 in AFHC. However, when applying Algorithm 1, there exists 50\% possibility that VNF $x$ on SFC $r$ is placed at different N-PoPs in $[t-1, t]$ (one time at $v_{1}$ and the other time at $v_{2}$ ). Then the migration cost of Algorithm 1 is positive and the ratio of Algorithm 1 with AFHC is infinite thus not bounded.

Targeting this drawback in the candidate path selection method in Algorithm 1, we design the online candidate path selection method. It migrates SFCs at time $t$ according to the method in Algorithm 1, when it is profitable for total cost reduction. On the other side, when the migration of an SFC is not profitable with large migration cost and little reduction of operating cost and congestion, the method can also place the SFC in the same way as that in time $t-1$.

The kernel of the online candidate path selection method is to define a criterion to decide whether an SFC should be migrated or not. Standing at time $t-1$, we define a parameter $\Pi_{r}(t)$ as the criterion to decide whether we migrate SFC $r$ between $t-1$ and $t$. The smaller $\Pi_{r}(t)$ is, the more necessary we need to migrate $r$ for total cost reduction. Otherwise, we place $r$ in the same way as that in $t-1$. Denote the total expected migration cost at $t$ is $\mathcal{E}_{t}$ when Algorithm 1 is applied on the result of AFHC. We then have $\mathcal{E}_{t}=\mathbb{E}\left[\sum_{r \in R} \sum_{x \in X_{r}} \delta_{r, x} \cdot\left|X_{r, x}^{v}(t)-\mathcal{X}_{r, x}^{v}(t-1)\right|\right]$. Denote the expected migration cost of SFC $r$ at time $t$ is $\mathcal{E}_{t, r}$. We have $\mathcal{E}_{t, r}=$ $\mathbb{E}\left[\sum_{x \in X_{r}} \delta_{r, x} \cdot\left|X_{r, x}^{v}(t)-\mathcal{X}_{r, x}^{v}(t-1)\right|\right]$. For the fractional result of AFHC, we define the total cost at $t$ as $C_{t}$ and the migration cost for SFC $r$ at $t$ as $C_{t, r}$. We then define $\Pi_{r}(t)=\frac{\mathcal{E}_{t}}{C_{t}+\sigma} \cdot \frac{\left|\mathcal{E}_{t, r}-C_{t, r}\right|}{\mathcal{E}_{t, r}+\sigma}$, where $\sigma$ is a very small positive number.

Here, $\frac{\mathcal{E}_{t}}{C_{t}+\sigma}$ denotes the ratio between the expected migration cost of Algorithm 1 and the total cost in the fractional solution of AFHC at time $t$. Large $\frac{\mathcal{E}_{t}}{C_{t}+\sigma}$ denotes the condition that the expected migration cost at $t$ is comparable to the total cost in AFHC. In this condition, SFC migration should be done carefully to avoid unnecessary migration cost. $\frac{\left|\mathcal{E}_{t, r}-C_{t, r}\right|}{\mathcal{E}_{t, r}+\sigma}$ indicates the gap between the expected migration cost for placing SFC $r$ in Algorithm 1 and the migration cost of $r$ in AFHC at time $t$. Large $\frac{\left|\mathcal{E}_{t, r}-C_{t, r}\right|}{\mathcal{E}_{t, r}+\sigma}$ represents the condition that there is a big difference between these two cost which is caused by the rounding manner of Algorithm 1. In this condition, the migration of SFC $r$ at time $t$ in Algorithm 1 can not decrease the overall cost efficiently. It is clear that $\Pi_{r}(t)$ is large only when both conditions hold. In this way, we perfectly target big $\Pi_{r}(t)$ on SFCs which should not be migrated between $t-1$ and $t$.

With $\Pi_{r}(t)$ calculated, we further use $\operatorname{Path}_{r}(t-1)$ to denote the chosen deployment of SFC $r$ at $t-1$. Path $(t-1)$ contains information of both VNF placement and flow routing. We then create a new set of candidate paths $\Omega_{r}^{\prime}(t)$ which contains both $\operatorname{Path}_{r}(t-1)$ and candidate paths for SFC $r$ at $t$ solved by Algorithm 1 . This means $\Omega_{r}^{\prime}(t)=\Omega_{r}(t) \cup$ Path $_{r}(t-1)$, where $\Omega_{r}(t)$ contains candidate paths $c p_{r, g}(t)$ from Algorithm 1 . We define $\frac{\Pi_{r}(t)}{\Pi_{r}(t)+1}$ as the probability of choosing $\mathrm{Path}_{r}(t-1)$ as deployment of SFC $r$ at time $t$. Therefore, the probability of choosing one candidate path from the rest of set $\Omega_{r}^{\prime}(t)$ is $\operatorname{Pr}\left(c p_{r, g}^{\prime}(t)\right)$ and

$$
\operatorname{Pr}\left(c p_{r, g}^{\prime}(t)\right)=\frac{1}{\Pi_{r}(t)+1} \cdot \operatorname{Pr}\left(c p_{r, g}(t)\right)
$$

With the candidate path sets and probabilities, the online candidate path selection method then select one candidate path for each SFC $r$ based on its probability as the placement of $r$ at time $t$.

With AFHC and the online candidate path selection method, the OCPS algorithm is presented and analyzed in Algorithm 2 in the following section.

\subsection{Online Candidate Path Selection Algorithm}

Algorithm 2 shows the detailed OCPS algorithm. The computational complexity of Algorithm 2 besides solving a convex problem is the same with that of Algorithm 1 as $O\left(|R||E|^{2}\right)$ for each time slot.

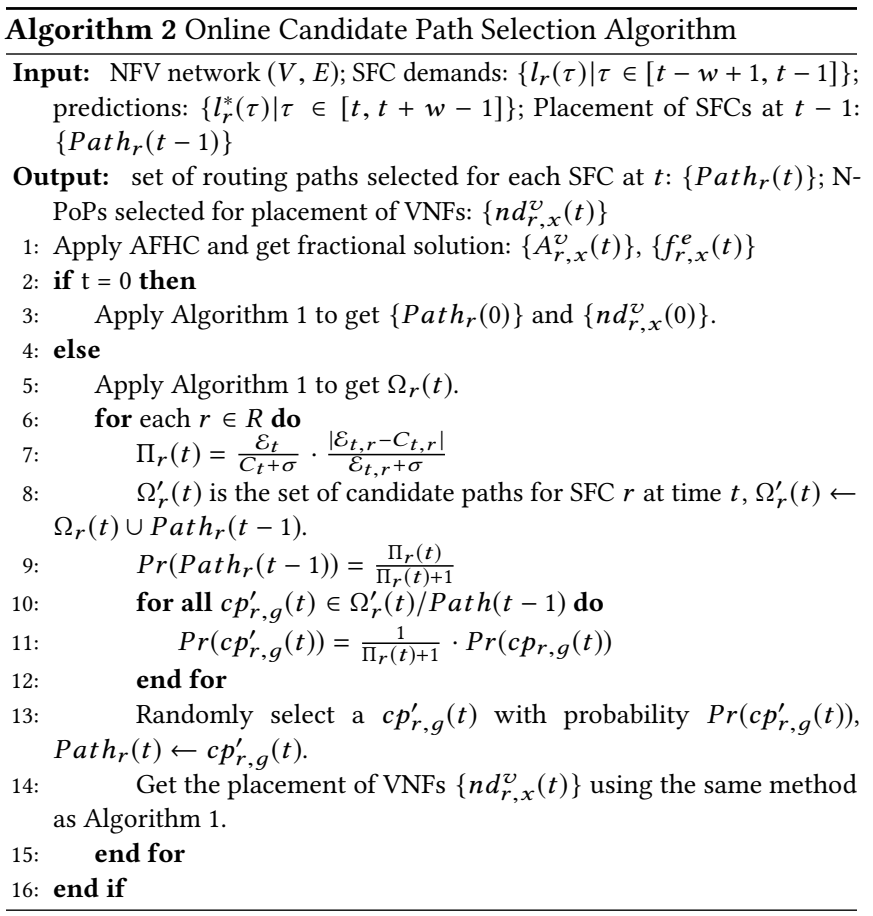

Based on the formulation of $\operatorname{Pr}\left(c p_{r, g}^{\prime}(t)\right)$, we analyze important features of Algorithm 2. First, when the expected migration cost at $t$ is small compared with the total cost in $\mathrm{AFHC}, \Pi_{r}(t)$ is much smaller than 1 . Thus, the probability of choosing $\operatorname{Path}_{r}(t-1)$ is negligible and $\operatorname{Pr}\left(c p_{r, g}^{\prime}(t)\right)$ converges to $\operatorname{Pr}\left(c p_{r, g}(t)\right)$. Therefore, the whole algorithm converges to Algorithm 1. In this case, the operating cost and the congestion cost take the majority of the total cost. According to similar proofs of Lemma 2.4, 2.5 and 2.6, we can prove that the sum of operating and congestion cost is bounded by $\log (|V|)$ times of the AFHC solution. With the proved competitive ratio of AFHC to the offline optimum, we can derive that the sum of the operating and congestion cost from Algorithm 2 has a competitive ratio of $\left[1+\Omega\left(\frac{1}{w}\right)\right] \cdot O(\log (|V|))$ to the offline optimal operating and congestion cost. Since the summation of two costs takes the majority of the overall cost under this situation, we consider the total migration cost is bounded by a constant. We then conclude that the overall cost is also bounded by $\left[1+\Omega\left(\frac{1}{w}\right)\right] \cdot O(\log (|V|))$ times of the offline optimum.

Second, when the expected migration cost at $t$ is large enough, yet the expected migration cost of SFC $r$ is similar with the AFHC result, $\Pi_{r}(t)$ is still much smaller than 1 . This indicates that SFC $r$ should be migrated for further reduction of the overall cost. In this 
case, Algorithm 2 will still deploy the SFC $r$ according to $\operatorname{Pr}\left(c p_{r, g}(t)\right)$ as that in Algorithm 1.

Third, when the migration cost is comparable with the total cost and there exists a big gap between the expected migration cost of SFC $r$ and the AFHC result, $\Pi_{r}(t)$ is much larger than 1 . In this case, placing SFC $r$ according to Algorithm 1 will introduce large migration cost without reducing enough operating and congestion cost. Therefore, Algorithm 2 prefers to leave the VNF placement and routing of SFC $r$ unchanged between $t-1$ and $t$.

\section{EVALUATION}

In this section, we first present the setup of simulations and then evaluate the performance of our SFC placement algorithms.

\subsection{Simulation Setup}

In this paper, we implement our simulations using Python and solve the linear programming problem with PuLP [16]. For the simulation setup, we refer to basic settings of the VNF platform on commercial servers in [13]. We consider each $\mathrm{N}-\mathrm{PoP}$ as one server with a single $\mathrm{CPU}$ of 6 cores. We use the utilization of $\mathrm{CPU}$ resources to represent the size of an SFC's demand (e.g., a VM takes up 0.6 core of a CPU). All detailed CPU utilization of SFCs comes from the CPU utilization of VMs in real traces from the Azure data. For simplicity of analysis, we assume that the ratio between the CPU utilization of an SFC and the amount of flow on this SFC is 1 . More generalized cases can be realized by simply adding a ratio parameter in the model.

The paper [13] does not involve information about links or networks connecting these servers and there exists a variety of possible network conditions in actual cases. In this way, we connect these servers with links of bandwidth uniformly distributed between 0 to the maximum bandwidth of the server's NIC port. The connection rate of each network is randomly picked between 0.3 to 0.8 . In this way, we can simulate the uncertainty of networks. Similarly, the CPU of an N-PoP may be occupied by tasks other than VNFs (e.g., control module, routing). So we also uniformly distribute available capacity of N-PoPs between 1 to 6 cores. We run our algorithms on a large number of these randomly generated networks for the generalized conclusion. In each network, there exist 20 to 30 servers with 40 to 80 SFCs. Values of parameters $\beta, \gamma$ and $\delta$ used in following simulations are all normalized by the average value of $\alpha$. Therefore, we can analyze the relations among the operating cost, the congestion cost and the migration cost when applying our algorithms without knowing their absolute values.

\subsection{Evaluation of the CPS Algorithm}

In this section, we present simulation results of Algorithm 1 (the CPS algorithm). For demands of SFCs, we randomly pick one time slot of CPU utilization of VMs from the real trace and assign them to the SFCs.

The first advantage of our algorithm is that it considers the operating cost and the network congestion simultaneously for overall cost reduction. In this way, Algorithm 1 prevents heavy network congestion when pursuing low operating cost. We verify this advantage in Fig. 2. The effect of reducing congestion is affected by relative values of congestion parameters $\beta$ and $\gamma$ which reflect service requirements and network conditions. We get the maximum congestion ratios on $\mathrm{N}$-PoPs and links in multiple simulations while increasing $\beta$ and $\gamma$ and present results in Fig. 2 (a). We observe that the congestion on
$\mathrm{N}$-PoPs decrease significantly when $\beta$ and $\gamma$ increase. This indicates that when congestion on N-PoPs weights more, our strategy further reduces the congestion for cost reduction. Moreover, we find that $\gamma$, the congestion parameter of links, also effects the congestion on $\mathrm{N}$ PoPs. If $\gamma$ increases together with $\beta$, congestion on $\mathrm{N}-\mathrm{PoPs}$ is further reduced. We can draw similar conclusions from results of congestion on links. Moreover, since load on links have no integral constraint with multi-path routing, the link congestion is sharply reduced as long as $\gamma>0$. Based on this figure, we conclude that our algorithm can reduce congestion on both $\mathrm{N}-\mathrm{PoPs}$ and links according to values of $\beta$ and $\gamma$.

Fig. 2 (b) shows the effect of total cost reduction of our algorithm while considering different types of congestion. All costs in the figure are normalized by costs of placing SFCs ignoring congestion, which means that the cost of congestion exists but the algorithm does not consider it. According to the figure, it is obvious that Algorithm 1 can reduce the total cost as long as congestion matters with positive $\beta$ and $\gamma$. In addition, the larger damage caused by congestion to the total profit, the better performance Algorithm 1 will have. Moreover, considering congestion on Both N-PoPs and links leads to the largest cost reduction.

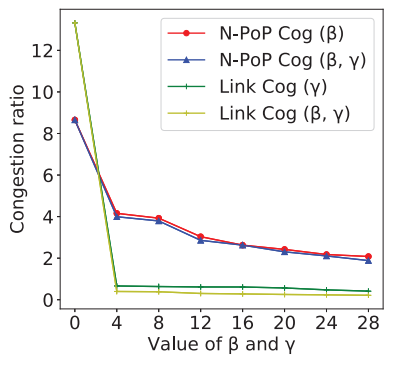

(a)

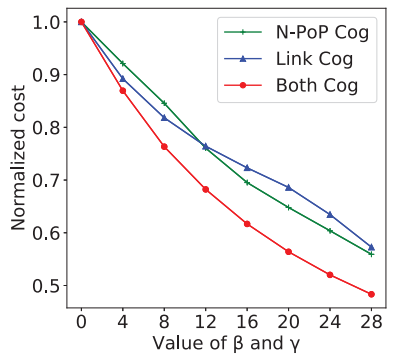

(b)
Figure 2: (a) presents average maximum congestion ratios on $\mathbf{N}$-PoPs and links when $\beta$ and $\gamma$ increase. The line marked $\mathbf{N}-$ PoP $\operatorname{Cog}(\beta)$ denotes the congestion ratios on $\mathbf{N}$-PoPs when $\beta$ changes with $\gamma=0$. The line marked $\mathrm{N}-\operatorname{PoP} \operatorname{Cog}(\beta, \gamma)$ denotes the congestion ratios on $\mathbf{N}-\mathbf{P o P s}$ when both $\beta$ and $\gamma$ increase. Other lines have similar definitions. (b) shows the total cost with increasing $\beta$ and $\gamma$ when different types of congestion are considered. All costs are normalized by costs of placing SFCs without considering the congestion. The line marked Both Cog means the total cost of SFC placement when congestion on both N-PoPs and links is considered.

The second advantage of our algorithm is that it jointly considers the VNF placement and flow routing for global optimization. In Fig. 3, we compare our algorithm with an SFC placement algorithm named $\mathrm{k}$-shortest paths. This algorithm first considers VNF placement to reduce the cost of VNF operating and congestion on N-PoP. It then manages the routing between adjacent VNFs by finding the first $\mathrm{k}$ shortest paths and distributing flows evenly on these paths. Fig. 3 (a) shows the congestion cost of Algorithm 1 and the k-shortest paths algorithm with different $\mathrm{k}$. We only consider $k$ no larger than 5 , because there are often less than 5 routing paths between N-PoPs in the majority of networks and it is inefficient to manage multiple routing paths with little amount of flow. The results of algorithms are normalized by results of the LP. Fig. 3 (b) shows the corresponding total cost of two algorithms. From both figures, we observe that the performance of $\mathrm{k}$-shortest paths improves with larger $\mathrm{k}$ but 
the improvement decreases as k grows. Furthermore, our algorithm always has lower congestion cost and total cost than the k-shortest paths algorithm even when $\mathrm{k}$ is large. This indicates the advantage of considering placement and routing jointly in our algorithm.

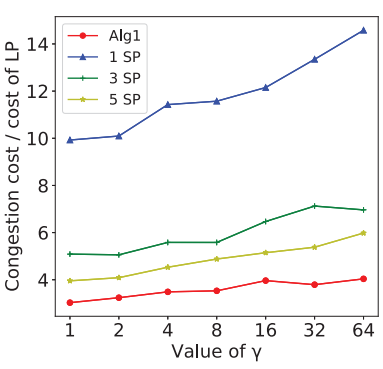

(a)

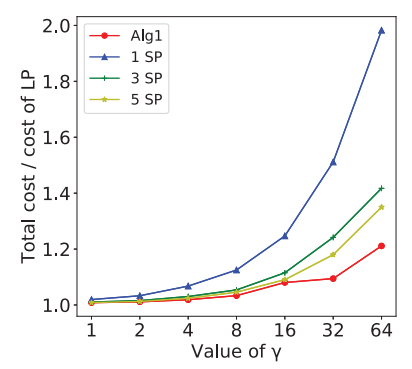

(b)
Figure 3: (a) shows the congestion costs on links normalized by results from the LP when $\gamma$ increases with different SFC placement strategies. (b) shows the corresponding result when counting the total cost. For all the simulations, $\beta=10$. Lines marked with $k$ SP represent results of the $k$-shortest paths algorithm with different $k$.

Algorithm 1 provides approximation results with distances to the optimal solutions. In Fig. 4, we validate the performance of Algorithm 1 in approaching the optimum. Fig. 4 (a) presents the cumulative distribution function (CDF) of total costs from Algorithm 1 normalized by costs from the LP in multiple simulations with $\beta=\gamma=10$. Results from the LP serve as the lower bounds of the optimal results from the original ILP problem. We observe that $90 \%$ of costs from Algorithm 1 are less than 1.05 times of costs from the LP and more than $99 \%$ are less than 1.1 times. In this way, we demonstrate the close distance between the performance of Algorithm 1 and the optimum.

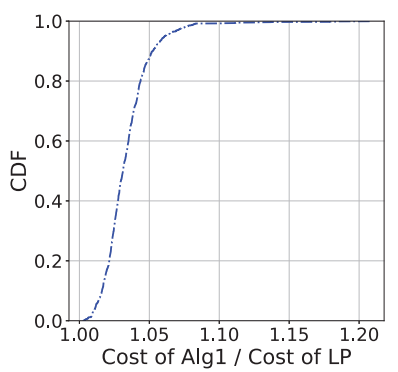

(a)

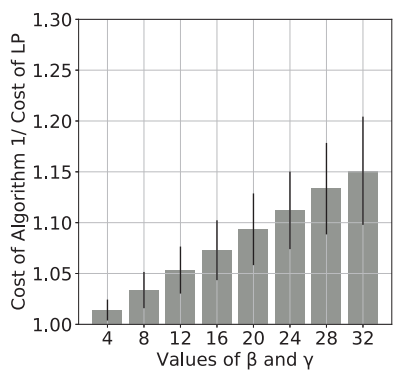

(b)
Figure 4: (a) shows the CDF of the costs from Algorithm 1 normalized by costs from the LP with $\beta=\gamma=10$. (b) shows the mean and standard deviation of results from Algorithm 1 normalized by the result from LP when $\beta$ and $\gamma$ increase.

We further find that values of $\beta$ and $\gamma$ affect the distance between Algorithm 1 and the optimal solution. Fig. 4 (b) presents the mean and standard variance of the costs from Algorithm 1 with increasing $\beta$ and $\gamma$. In the figure, all values are normalized by costs from the LP. We observe that both mean and variance of the normalized costs are very small when $\beta$ and $\gamma$ are relatively small. When $\beta$ and $\gamma$ grow, mean and variance also grow correspondingly. This indicates that larger congestion parameters lead to larger distances between Algorithm 1 and the optimum. However, the performance of Algorithm 1 is still close to the optimal solution and stays in the proved bound even with large $\beta$ and $\gamma$. In this way, the theoretical bound proved in Section 2 is validated by simulation results.

With results above, Algorithm 1 is verified to be effective in reducing the operating cost and the congestion cost while jointly placing VNFs and routing flows. We then present simulation results of the online SFC placement algorithm dealing with fast scaling demands.

\subsection{Evaluation of the OCPS Algorithm}

We simulate Algorithm 2 (the OCPS algorithm) using SFC demands from the real trace. Each time slot in the real trace expands for 5 minutes and the total time span $T$ is 50 . All results shown below are average values over multiple simulations.

As mentioned in Section 3.2, AFHC is extremely suitable for the online SFC deployment strategy. To verify this conclusion, we compare the results of Algorithm 2 using AFHC and the receding horizon control (RHC) [14] [3]. In Fig. 5 (a), we show their competitive ratios with increasing prediction window size. The competitive ratios are between results of Algorithm 2 and the (fractional) solutions of the offline convex problem, which serve as the lower bounds of the offline optimum (integral). Getting the offline optimal results involves solving large-scale NP-hard problems with integer constraints which are too time-consuming to afford. It is clear that competitive ratios of Algorithm 2 with both AFHC and RHC decrease with larger window size and the competitive ratios with AFHC is always much smaller than that with RHC. This indicates that AFHC contributes to better performance than RHC for Algorithm 2.

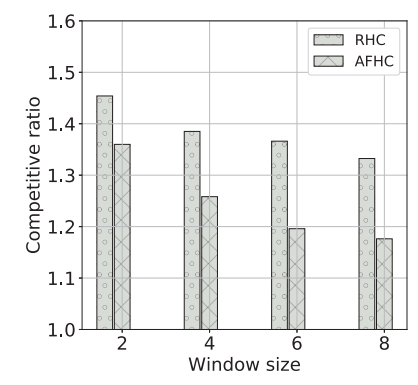

(a)

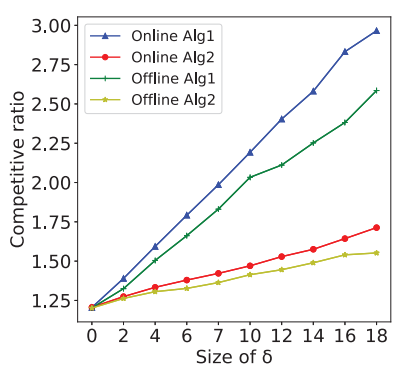

(b)
Figure 5: (a) shows competitive ratios of Algorithm 2 using AFHC and RHC with different window sizes. (b) shows the competitive ratios of the offline Algorithm 1,2 and the online Algorithm 1, 2 with a window size of 4 .

In Fig. 5 (b), we present the comparison of Algorithm 1 and Algorithm 2. Both algorithms apply AFHC on the relaxed OCO problem then use different candidate path selection methods to yield SFC placement. It is clear that the online results (with unknown future demands) of both algorithms are close to their corresponding offline results (with all demands known along the time span). This indicates the effectiveness of AFHC. We then observe that, with $\delta$ increasing, the competitive ratios of both online algorithms deviate from the $\mathrm{x}$-axis. This deviation is mainly caused by the unbounded migration cost demonstrated in Section 3.3. However, the ratios of Algorithm 2 deviate much slower than that of Algorithm 1. In this way, we conclude that Algorithm 2 outperforms Algorithm 1 in approaching the optimum. Thus, the goal of designing Algorithm 2 is achieved. Furthermore, We observe that Algorithm 2 stays close to the lower bound of the offline optimum even with large $\delta$. And Algorithm 2 is even closer to the actual offline optimal (integral) solutions than 
the lower bounds. Therefore, simulations validate that the performance of Algorithm 2 is close to the offline optimum which makes it a promising candidate for congestion-aware online SFC placement and load balancing.

\section{RELATED WORK}

In recent years, methods have been proposed for deploying SFCs with different optimization objectives. Luizelli et al. [11] formulate an Integer Linear Programming (ILP) model that provides an optimal solution to minimize the number of VNF instances (VMs) used during the placement of SFCs. Sang et al. [18] propose two greedy algorithms for the same optimization goal with an approximation ratio of $(1-o(1)) \ln (m)+2$, where $m$ is the number of flows. Mehraghdam et al. [15] use a context-free language to formalize the chaining of network functions. For the purpose of reducing the operating cost of VNFs, Moens et al. [17] propose a model called VNF-P for VNF resource allocation which focuses on a hybrid scenario where VNFs are provided by both dedicated physical hardware and virtualized service instances. However, above papers have not considered congestion problems in VNF networks.

For the network congestion, Sekar et al. propose a model named $\mathrm{CoMb}$ that explores consolidation opportunities to reduce network provisioning cost and load skew in [19]. Ma et al. formulate the Traffic Aware Placement of interdependent middleboxes problem in [12]. They consider traffic changing effects of the middleboxes to avoid resource contention and congestion. Carpio et al. propose optimization models and heuristic algorithms to balance the load on links using replication in [1]. Nevertheless, to the best of our knowledge, no work has been done on jointly reducing the operating cost of VNFs and the congestion on N-PoPs and links.

On the other hand, several quality schemes have been proposed to deal with the online pattern of VNF demands. Zhang et al. fuse online learning and online optimization to provide a proactive VNF provision with Multi-timescale cloud resources in [21]. The paper mainly focuses on online scaling and distribution of VNFs without detailed placement and routing. Fei et al. [5] propose a scheme to predict upcoming SFC demands using online learning. The VNF placement and routing are then processed based on the prediction. Yet, this paper process VNF placement and flow routing separately and leave space for further improvement with joint placement and routing. Guo et al. [7] propose an adaptive online algorithm to place and route VNFs whenever a new request comes. However, they reduce the complexity by predefining sets of routing paths at the cost of optimality. The network congestion can be further reduced with more choices of VNF placement and flow routing. In addition, the above literature does not allow VNF migration, which is a key concern in our paper.

\section{CONCLUSION}

In this paper, we study the problem of joint optimization of the SFC placement and flow routing to minimize the operating cost and network congestion simultaneously in VNF networks. Due to the NP-hardness of the problem, we first propose an SFC placement algorithm to solve the offline problem with bounded approximation ratio. To handle the lack of future information and fast demand fluctuation, we further propose an online algorithm. Extensive simulations validate the effectiveness of both algorithms. In the future, we plan to investigate the performance of other online optimization algorithms and how to select the best algorithm in an online manner.

\section{ACKNOWLEDGMENTS}

This research work was supported in part by the U.S. National Science Foundation under grant numbers CCF-1526162 and CCF-1717731.

\section{REFERENCES}

[1] Francisco Carpio, Samia Dhahri, and Admela Jukan. 2017. VNF placement with replication for Loac balancing in NFV networks. In Communications (ICC), 2017 IEEE International Conference on. IEEE, 1-6.

[2] Niangjun Chen, Anish Agarwal, Adam Wierman, Siddharth Barman, and Lachlan L.H. Andrew. 2015. Online Convex Optimization Using Predictions. In Proceed ings of the 2015 ACM SIGMETRICS International Conference on Measurement and Modeling of Computer Systems. IEEE, 191-204.

[3] Niangjun Chen, Joshua Comden, Zhenhua Liu, Anshul Gandhi, and Adam Wierman 2016. Using predictions in online optimization: Looking forward with an eye on the past. ACM SIGMETRICS Performance Evaluation Review 44, 1 (2016), 193-206.

[4] GSNFV ETSI. 2013. Network functions virtualisation (nfv): Architectural framework. ETsI Gs NFV 2, 2 (2013), V1.

[5] Xincai Fei, Fangming Liu, Hong Xu, and Hai Jin. 2018. Adaptive vnf scaling and flow routing with proactive demand prediction. In IEEE INFOCOM 2018-IEEE Conference on Computer Communications. IEEE, 486-494.

[6] Hao Feng, Jaime Llorca, Antonia M Tulino, Danny Raz, and Andreas F Molisch. 2017. Approximation algorithms for the NFV service distribution problem. In INFOCOM 2017-IEEE Conference on Computer Communications, IEEE. IEEE, 1-9.

[7] Linqi Guo, John Pang, and Anwar Walid. 2018. Joint Placement and Routing of Network Function Chains in Data Centers. In IEEE INFOCOM 2018-IEEE Conference on Computer Communications. IEEE, 612-620.

[8] Kai Han, Zhiming Hu, Jun Luo, and Liu Xiang. 2015. RUSH: Routing and scheduling for hybrid data center networks. In Computer Communications (INFOCOM), 2015 IEEE Conference on. IEEE, 415-423.

[9] Jian-Jhih Kuo, Shan-Hsiang Shen, Hong-Yu Kang, De-Nian Yang, Ming-Jer Tsai, and Wen-Tsuen Chen. 2017. Service chain embedding with maximum flow in software defined network and application to the next-generation cellular network architecture. In INFOCOM 2017-IEEE Conference on Computer Communications, IEEE. IEEE, 1-9.

[10] Minghong Lin, Zhenhua Liu, Adam Wierman, and Lachlan LH Andrew. 2012 Online algorithms for geographical load balancing. In Green Computing Conference (IGCC), 2012 International. IEEE, 1-10.

[11] Marcelo Caggiani Luizelli, Leonardo Richter Bays, Luciana Salete Buriol, Marinho Pilla Barcellos, and Luciano Paschoal Gaspary. 2015. Piecing together the NFV provisioning puzzle: Efficient placement and chaining of virtual network functions. In Integrated Network Management (IM), 2015 IFIP/IEEE International Symposium on. IEEE, 98-106.

[12] Wenrui Ma, Oscar Sandoval, Jonathan Beltran, Deng Pan, and Niki Pissinou. [n d.]. Traffic Aware Placement of Interdependent NFV Middleboxes.

[13] Joao Martins, Mohamed Ahmed, Costin Raiciu, Vladimir Olteanu, Michio Honda, Roberto Bifulco, and Felipe Huici. 2014. ClickOS and the art of network function virtualization. In Proceedings of the 11th USENIX Conference on Networked Systems Design and Implementation. USENIX Association, 459-473.

[14] David Q Mayne and Hannah Michalska. 1990. Receding horizon control of nonlinear systems. IEEE Transactions on automatic control 35, 7 (1990), 814-824.

[15] Sevil Mehraghdam, Matthias Keller, and Holger Karl. 2014. Specifying and placing chains of virtual network functions. In Cloud Networking (CloudNet), 2014 IEEE $3 r d$ International Conference on. IEEE, 7-13.

[16] Stuart Mitchell and Iain Dunning. 2011. PuLP: a linear programming toolkit for python. (2011)

[17] Hendrik Moens and Filip De Turck. 2014. VNF-P: A model for efficient placement of virtualized network functions. In 10th International Conference on Network and Service Management (CNSM). 418-423.

[18] Yu Sang, Bo Ji, Gagan R Gupta, Xiaojiang Du, and Lin Ye. 2017. Provably Efficient Algorithms for Joint Placement and Allocation of Virtual Network Functions. In Computer Communications, IEEE INFOCOM 2017-The 36th Annual IEEE International Conference on. IEEE.

[19] Vyas Sekar, Norbert Egi, Sylvia Ratnasamy, Michael K Reiter, and Guangyu Shi 2012. Design and implementation of a consolidated middlebox architecture. In Proceedings of the 9th USENIX conference on Networked Systems Design and Implementation. USENIX Association, 24-24.

[20] Xiaojun Shang, Zhenhua Li, and Yuanyuan Yang. 2018. Placement of Highly Available Virtual Network Functions Through Local Rerouting. In 2018 IEEE 15th International Conference on Mobile Ad Hoc and Sensor Systems (MASS). IEEE, 80-88.

[21] Xiaoxi Zhang, Chuan Wu, Zongpeng Li, and Francis CM Lau. 2017. Proactive vnf provisioning with multi-timescale cloud resources: Fusing online learning and online optimization. In IEEE INFOCOM 2017-IEEE Conference on Computer Communications. IEEE, 1-9. 